\title{
Parallel multichannel architecture for surface plasmon resonance sensors
}

\author{
R. Kasztelanic \\ kasztel@igf.fuw.edu.pl
}

University of Warsaw, Department of Physics, ul. Pasteura 7, 02-093 Warsaw, Poland

The paper deals with an optical sensor based on the phenomenon of surface plasmon resonance. It proposes a new geometry of the measurement head which allows for measurements with both the change of the incident light angle and the change of the wavelength. The sensors proposed can also be used in parallel configurations, where they increase the functionality of the setup, allow for a greater precision of measurement, and eliminate such distracting factors as temperature change. The article presents the results of computer analyses of the sensor proposed, as well as the results of its experimental realization.

[DOI: http://dx.doi.org/10.2971/jeos.2012.12038]

Keywords: Surface plasmon resonance, optical sensor, chemical sensor, biosensor, multisensing SPR

\section{INTRODUCTION}

Optical sensors are based on various phenomena occurring when light interacts with matter. One of such phenomena is surface plasmon resonance (SPR) [1]. It occurs when conditions for resonance of an electromagnetic wave propagating along the metal-dielectric boundary are fulfilled. Due to the fact that resonance occurs only for a narrow range of parameters, sensors based on this phenomenon are characterized by high sensitivity. In the case of SPR, the main parameter influencing the conditions of resonance is the refraction index of the examined substance. In order to measure a different parameter such as concentration, or biospecific interactions, the sensor will be designed in a way which combines the change of the parameter with the change of the refraction index.

So far, the methods for establishing the refraction index (RI) have consisted in measuring the intensity of the electromagnetic wave near the resonance [2], measuring its intensity depending on the angle of the incident light [3], or its intensity depending on the wavelength [4]. Other types of setups use the phase change [5], the change of light polarization [6], or the hybrid differential interrogation [7] method. However, it turns out that with the use multi-canal setups [8], it is possible to simultaneously measure the change of the angle and the wavelength [9], as well as to take measures.

This paper presents a new architecture of the SPR sensor based on a prism in an attenuated total reflection configuration (ATR). This architecture allows for miniaturizing the SPR sensor and using angular and wavelength modulation simultaneously. It increases the measurement precision and partly eliminates factors distorting the measurement, such as temperature. It also allows for measurements in a wide RI range and a wide range of angles without any moving or rotating elements. With the new architecture, it is possible to build multichannel setups in both the parallel and series configurations. The paper presents the results both the computer simulations

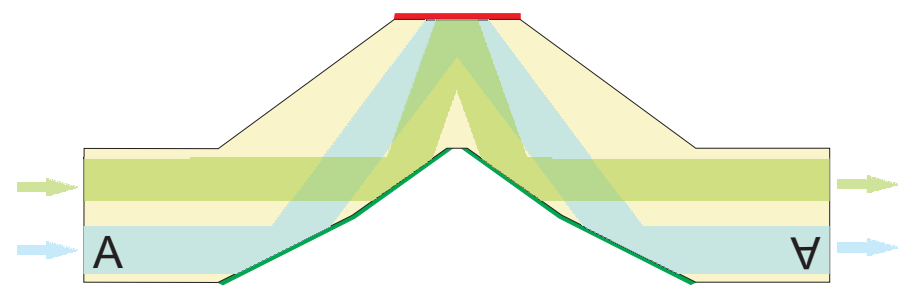

FIG. 1 Simplified version of the SPR sensor head. The red line mark the part of the sensor where the SPR takes place. The green line marks the reflecting surfaces, whose aim is to form the beam.

and the experimental realization of the novel SPR sensor architecture.

\section{SENSOR DESIGN}

In this article a new SPR head is proposed and analyzed. The head allows for the RI measurement with both the angular modulation and the wavelength modulation without intervening with the head of the SPR setup. The setup proposed works in a configuration based on the Kretschmann geometry, where the excitation of surface plasmons is performed by the attenuated total reflection (ATR) [1]. The simplified schemes of the SPR head proposed are presented in Figure 1 [10]. The scheme shows only two measurement channels, but the architecture allows for using a number of them.

In the proposed head (Figure 1), both the incident and the output beam take the form of a plane wave. The first system of mirrors (green lines) forms the incident wave into several beams, each of them lighting at a different angle on the plane where SPR takes place (red line). The values of the lighting angles of the beams depend on the materials from which the sensor is fabricated, the range of the refraction index to be measured by the sensor, as well as the length of the electro- 
magnetic wave used. The second symmetrical system of mirrors finally forms the beam into the plane output wave. The advantage of this setup is the flat metal-dielectric boundary where SPR takes place, which allows for the use of exchangeable working elements. Additionally, in this setup all the light reflections within the head take place in the conditions of total internal reflection, which does not require a special spattering of the mirrors and considerably simplifies the fabrication process of the element. A disadvantage is that it is not possible to obtain the continuous change of the lighting angle of the incident beam. Also, the image registered at the output of the setup is not continuous, due to the fact that the surface of the mirrors forming the beam takes the form of steps.

A characteristic feature of the setup is the fact that the beam is reversed in each of the channels (symbolically presented with the letter A in Figure 1) at the input and the output of the setup. This feature does not influence the quality of the work of the setup.

\section{COMPUTER SIMULATION}

The aim of the computer simulation carried out was to check the influence of various parameters of the SPR head the on the preciseness of the results obtained. The parameters used were: a different the number of the measurement channels, the change of the wave length and the use of the parallel and series architecture of the sensor. In the simulations it was assumed that the SPR head was made of PMMA with the refraction index $n=1.49(\lambda=632.8 \mathrm{~nm})$ and the active area was covered in a $50 \mathrm{~nm}$ thick silver layer. The range of the work of the sensor, which is the range of the coefficients measured, was set between 1.31 and 1.36 .

For the parameters defined this way, the range of the incident angles varies from about 67 to 77 degrees, because for such angles it is possible to obtain the minimum intensity of the reflected light. Figure 2 presents the correlation of the reflection and the incident angle for two wavelengths. The color region shows an arbitrary choice of angles from 67 to 77 degrees, for which measurements are to betaken. Figure 3 shows an example of the simulation results obtained for the proposed SPR head with 11 measurement channels. The figure also shows a scheme of the measured RI value of the examined substance. The measurement consists in fitting the SPR curve to the results obtained with the change of one parameter only, which is the RI. The main parameter influencing the preciseness of the results obtained is the number of the measurement channels of the SPR head. In this case, the number of channels is understood as the number of various angles for which a single measurement can be taken.

In order to estimate the sensitivity of the setup, it was checked how the minimal change of the refraction index $(\Delta n)$ of the examined substance changes the results on the detector. This sensitivity depends on the number of channels and the dynamic range of the detector, as well as on the RI value, due to the discreteness of the measurement. Theoretically, there should be as many channels as possible; however, the geometry of each of the heads proposed limits the number of the

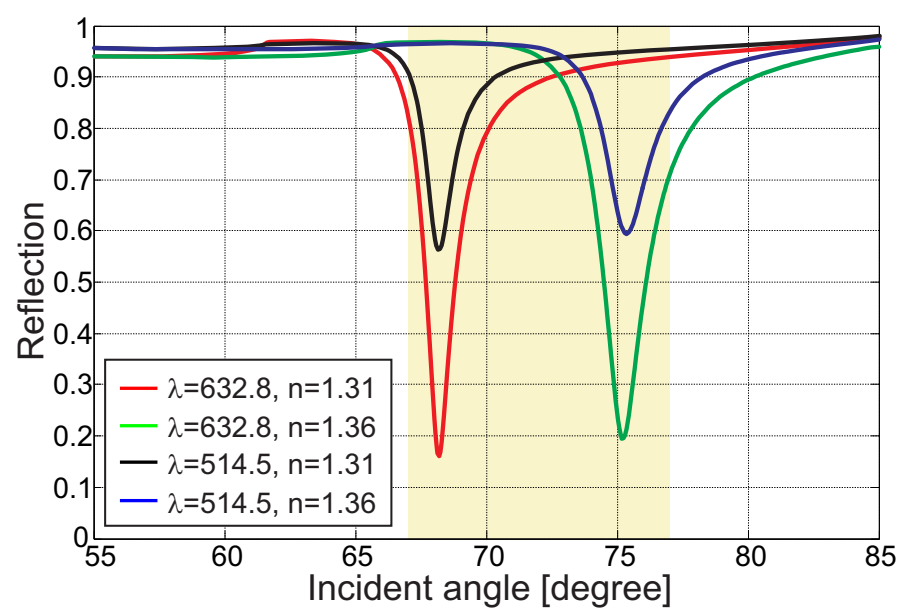

FIG. 2 The dependence of the reflection on the incident angle on the surface where SPR takes place. The color area between 67 and 77 degrees is the range of work of the SPR sensor.

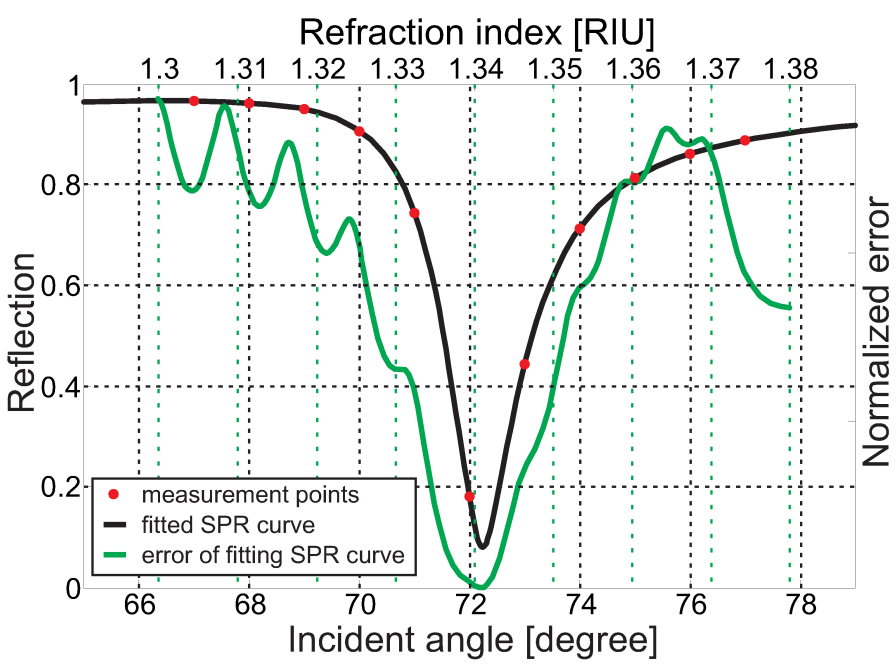

FIG. 3 The exemplary results for the SPR head with 11 measurement channels for immersion liquid $n=1.341$.

discrete angle values. Taking into consideration the technological possibilities of fabricating the SPR heads, the width of a single channel should not be too small, since then the precision of the element fabrication may considerably influence of the quality of the results obtained. So, the ultimate number and size of the channels depend on the size of the SPR head and the technology of its fabrication. For an 11-channel sensor with an 8-bit detector it is possible to measure the refraction index at the level of $3 \times 10^{-5}$ RIU. Increasing the number of channels to 20 and the use of a 10-bit detector gives the theoretical sensitivity of the setup at the level of $4 \times 10^{-6}$ RIU.

The biggest advantage of the present SPR sensor is the possibility of using of the same head to measure several different wavelengths, which increases the sensitivity of the setup. For this purpose a supercontinnum source can be used [11]. The data obtained from computer simulations show that for a head with 11 channels and an 8-bit dynamic range of the detector, each doubling of the number of the wavelengths used increases the sensitivity of the setup 1.5 times, on the average. 




FIC. 4 A complex SPR head in parallel configuration with a separated layer where SPR takes place.

It has also been tested how the lack of precision in the fabrication of the element influences the quality of measurements. It seems that the flaws which potentially cause most measurement errors are those connected with the imprecision of the element geometry. Such flaws are, however, easy to eliminate through independent measurements of geometry and the right correction when fitting the curve to the measurement data. Yet another factor influencing the measurement quality is the thickness of the metal layer. The imprecision in sputtering in the range of $1 \mathrm{~nm}$ results in the measurement error of $8 \times 10^{-5}$ RIU. Also this imprecision can be partially eliminated thanks to referential measurement.

\section{PARALLEL CONFIGURATION}

The number of the channels available can be increased without changing the size of the SPR head and the size of a single channel. It can be done through parallel joining of several SPR heads, each of them containing channels tilted at a different angle. The problem of penetration of the light wave between the heads can be eliminated thanks to a reflective layer placed between every two heads. An example of such a setup containing three SPR heads is presented in Figure 4. In this configuration, it is possible to use a different type of metal or biomolecular coating in each of the heads. The material of each head can also be different, which further increases the capabilities of the setups discussed. It is also possible to separate the active part of the head where SPR takes place from the part with channels, as presented in Figure 4 . The separation allows for reusing the heads.

Additionally, the parallel configuration compensates for the influence of temperature, which is extremely important for measurements in the range of $10^{-6}$ RIU $[12,13]$. Taking into consideration that the change of temperature results not only in the change the RI of the examined substance, but also the RI of the dielectric and the metal of which the head is fabricated, temperature compensation can be obtained through the use of several SPR heads, made of different materials. The temperature dependence of the RI of these materials is different. Such a setup is temperature-insensitive without any additional elements, or temperature stabilizing devices. This simplifies the sensor and makes it more flexible and cost-effective. Thanks to the compact build, the setup with parallel configuration is characterized with low

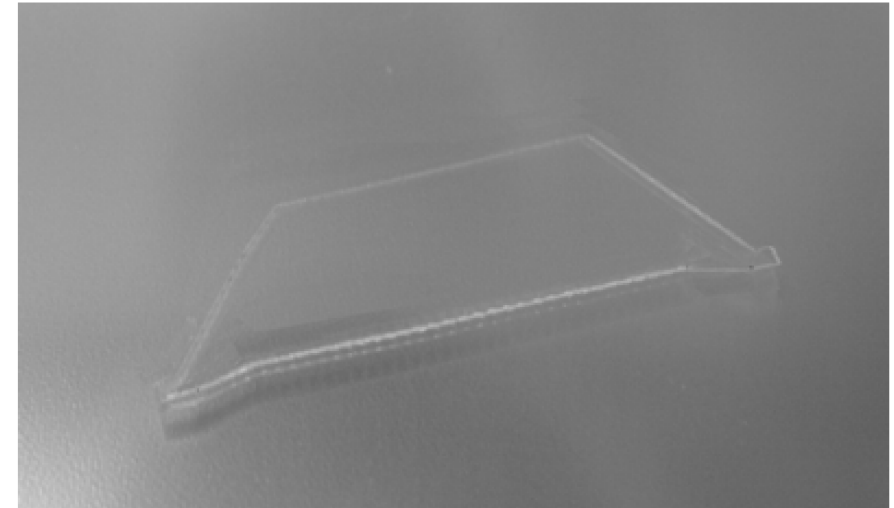

(a)

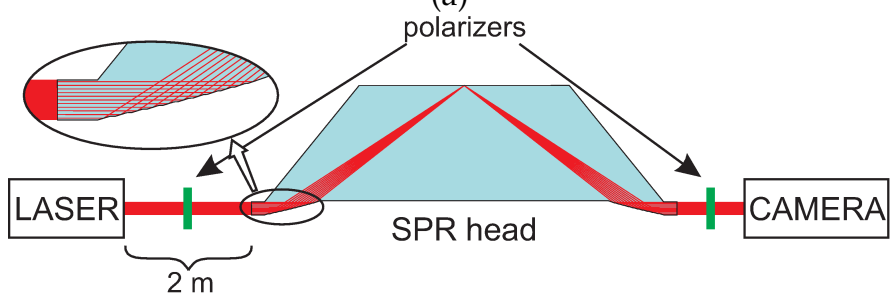

(b)

FIG. 5 The SPR head fabricated in the deep proton lithography technology (a). A scheme of the measurement setup (b).

temperature inertia.

\section{EXPERIMENTAL REALIZATION}

The choice of the version of the SPR heads depends on the parameters of the martial and the range, as well as the assumed sensitivity of the RI measurement. Additionally, there may be technological limitations as to the materials used.

In the present experiment, the SPR head fabricated in deep proton lithography method [14, 15] was used. This technology allows for producing elements of thickness above $1 \mathrm{~mm}$. In our case a proton beam of 7,4 MeV was used, which theoretically allowed for creating an element over $500 \mu \mathrm{m}$ thick. However, due to the shape of the side walls [15] where SPR takes place, this thickness was reduced to $250 \mu \mathrm{m}$. Protons were formed into a narrow beam with the use of a mask with circular hole of $50 \mu \mathrm{m}$. The beam cut the required shape from $250 \mu$ m-thick PMMA - poly(methyl methacrylate). The SPR head (Figure 5(a)) was obtained thanks to chemical etching.

The element fabricated measured $16 \times 5 \mathrm{~mm}$. Such a size was chosen due to the limitations of the technology used, as well as the necessity of the further manual adjustment of the measurement setup. In the next step the element was metalized with a $2 \mathrm{~nm}$ adhesive layer of chromium and 50 $\mathrm{nm}$ layer of silver on the surface where SPR takes place. The remaining walls were also coated in layers of these metals, apart from the places of the penetration of the input and the output beam. The thickness of these metal layers was not measured or optimized. In order to reduce the cost of the sensor, a single element produced in the proton lithography 
technology can be replicated by the molding process [14]. What is important, for elements fabricated in the proton lithography technology, stress or induced birefringence which could change the refraction index of the SPR head material and influence the precision of the measurement have not been noticed.

The SPR head was placed in a measurement setup consisting of an He-Ne laser of $3 \mathrm{~mW}$, two linear polarizers and an 8-bit dynamic range camera (Figure 5(b)). The laser beam was not specially formed into the plane wave. An approximated plane beam was obtained by placing the laser about $2 \mathrm{~m}$ from the measurement head.

The measurement was made by placing a drop of liquid to be examined (known RI) onto the SPR head and registering the image on the camera. An example of an image registered on the camera for a drop of refractive index liquid (Cargille Labs, $n=1.3520 \pm 0.0002$ ) is shown in Figure 6(a). The areas marked in red are the ones where measurement was taken for each of the channels. The average value of light intensity from each of the areas is the basis for establishing the RI value. Establishing the RI value is done through fitting the SPR curve to the experimental data (presented in Figure 6(b)). In the case of the measured liquid, the RI value was $1.35185 \pm 0.00007$. A similar comparability of results was obtained for a different wavelength and for several kinds of immersive liquids and water, whose RI was within the range of 1.31 to 1.36 . The measurement error was estimated at $7 \times 10^{-5}$ RIU for a single measurement, thanks to referential measurements. The error can be considerably reduced, however, with the use of parallel configuration and more than one wavelength for each measurement.

\section{CONCLUSIONS}

The paper presented a proposal and proof of principle experimental realization of a new configuration of a sensor for measuring the refraction index with the use of the phenomenon of the surface plasmon resonance. The main advantage of the solution proposed is the possibility of using the same head for measuring both the angle function and the wavelength function. This increases the functionality of the setup and allows for higher precision of the measurement. An additional advantage of the setup is its scalability, consisting in the use of several heads in a parallel configuration, which again allows for higher precision of the measurements, and compensates for the influence of the temperature.

The proposed configuration of sensors opens up a possibility of scaling the setup, depending on the measurement preciseness needed. Taking measurements with the change of the wavelength and/or the change of the incident light angle allows for a better flexibility and adjustment to the character of the concrete measurement taken. The setup is also more flexible and cost-effective due to the wider choice of its elements.

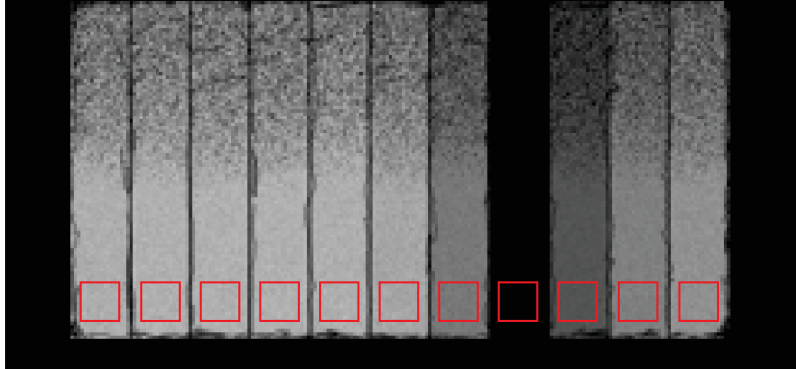

\begin{tabular}{|l|l|l|l|l|l|l|l|l|l|l|}
\hline 207 & 208 & 208 & 206 & 202 & 187 & 130 & 42 & 128 & 165 & 182 \\
\hline
\end{tabular}

(a)

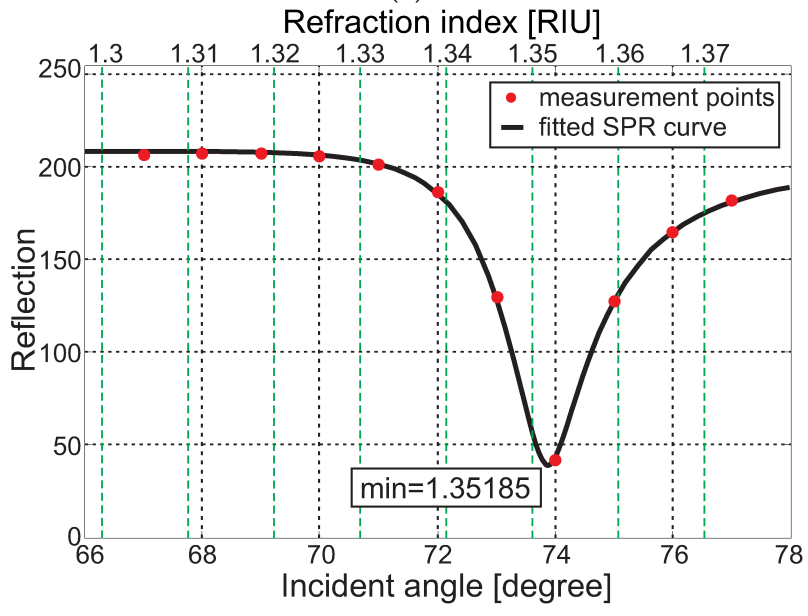

(b)

FIG. 6 Experimental results. The result of the RI measurement for immersion liquid $\mathrm{n}=1.3520$ (a) and fitting the SPR curve to establish the RI value (b).

\section{References}

[1] J. Homola, "Present and future of surface plasmon resonance biosensor," Anal. Bioanal. Chem. 377, 528-539 (2003).

[2] C. Nylander, B. Liedberg, and T. Lind, "Gas detection by means of surface plasmon resonance," Sensors Actuator. 3, 79-88 (1982).

[3] K. Matsubara, S. Kawata, and S. Minami, "Optical chemical sensor based on surface plasmon measurement," Appl. Optics 27, 1160-1163 (1988).

[4] L. M. Zhang, and D. Uttamchandani, "Optical chemical sensing employing surface plasmon resonance," Electron. Lett. 23, 1469-1470 (1988).

[5] P. Markowicz, W. Law, A. Baev, P. Prasad, S. Patskovsky, and A. Kabashin, "Phase-sensitive time-modulated surface plasmon resonance polarimetry for wide dynamic range biosensing," 0 pt. Express 15, 1745-1754 (2007).

[6] A. Kruchinin, and Y. Vlasov, "Surface plasmon resonance monitoring by means of polarization state measurement in reflected light as the basis of a DNA probe biosensor," Sensor Actuat. B - Chem. 30, 77-80 (1996).

[7] K. Wang, Z. Zheng, Y. Su, Z. Wang, L. Song, and J. Zhu, “Hybrid differential interrogation method for sensitive surface plasmon resonance measurement enabled by electro-optically tunable SPR sensor," Opt. Express 17, 4468-4478 (2009).

[8] C. E. H. Berger, T. A. M. Baumer, R. P. H. Kooyman, and J. Greve, "Surface plasmon resonance multisensing," Anal. Chem. 70, 703-706 (1998).

[9] S. R. Karlsen, K. S. Johnston, R. C. Jorgenson, and S. S. Yee, “Simultaneous determination of refractive index and absorbance spectra 
of chemical samples using surface plasmon resonance," Sensor Actuat. B - Chem. 25, 747-749 (1994).

[10] R. Kasztelanic, "Surface plasmon resonance sensors - novel architecture and improvements," Opt. Appl. 41, 145-155 (2011).

[11] R. Buczyński, D. Pysz, R. Stẹpień, A. J. Waddie, I. Kujawa, R. Kasztelanic, M. Franczyk, and M. R. Taghizadeh, "Supercontinuum generation in photonic crystal fibers with nanoporous core made of soft glass," Laser Phys. Lett. 8, 6, 443-448 (2011).

[12] M. Piliarik, and J. Homola, "Surface plasmon resonance (SPR) sensors: approaching their limits?," Opt. Express 17, 16505-16517 (2009).
[13] A. Naimushin, S. Soelberg, D. Bartholomew, J. Elkind, and C. Furlong, "A portable surface plasmon resonance sensor system with temperature regulation," Sensor Actuat. B - Chem. 96, 253-260 (2003).

[14] M. Kufner, S. Kufner, Micro-optics and lithography (VUB press, Brussels, 1997).

[15] R. Kasztelanic, "Multilevel structures in deep proton lithography," P. Soc. Photo-Opt. Ins. 07(01), 013006 (2008). 